Revista Eletrônica de Farmácia

Eletronic Journal of Pharmacy

ISSN 1808-0804

doi $10.5216 /$ ref.v16i0.47950

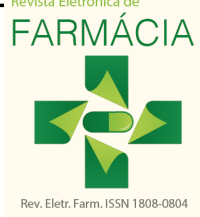

Artigo Original

\title{
Nutracêuticos: aspectos legais e científicos
}

\section{Nutraceuticals: legal and scientifics aspects}

\section{Nutracéuticos: aspectos legales y científicos}

\author{
MACHADO, Gabriela ${ }^{1}$. PUTON, Bruno Furini ${ }^{2}$. BERTOL, Charise ${ }^{34 *}$ \\ ${ }^{1}$ Especialização em Farmacologia Clínica, Curso de Farmácia, Instituto de Ciências Biológicas, Universidade de Passo Fundo - \\ ICB/UPF. ${ }^{2}$ Departamento de Biologia e Farmácia, Universidade de Santa Cruz do Sul - Unisc. ${ }^{3}$ Curso de Farmácia, Instituto de \\ Ciências Biológicas, Universidade de Passo Fundo - ICB/UPF. ${ }^{4}$ Programa de Pós-Graduação em Envelhecimento Humano, \\ Universidade de Passo Fundo - PPGEH/UPF. \\ *charise@upf.br
}

Resumo. Introdução: Os nutracêuticos vêm se destacando por apresentarem diversos benefícios à saúde, prevenindo e auxiliando no tratamento de doenças. Os ácidos graxos poli-insaturados (AG), os carotenoides, os flavonoides e as isoflavonas estão entre os mais consumidos. Objetivo: Este estudo objetivou pesquisar definições, regulamentações, comprovações sobre eficácia, tratamento e reações adversas dos nutracêuticos. Resultados: Os AG apresentam propriedades cicatrizantes e de regeneração tecidual, imunorreguladoras e anti-inflamatórias, e, reguladoras do perfil lipídico. O betacaroteno previne câncer, doenças cardiovasculares e renais, dentre outras, por outro lado, em fumantes pode aumentar a incidência de câncer de pulmão. Entre os flavonoides, a quercetina se destaca pelos seus efeitos antioxidantes, protetor dos sistemas renal, cardiovascular e hepático, porém deve ser evitada por pacientes hipotensos. As isoflavonas são uteis nos sintomas da menopausa, incluindo a depressão. Conclusão: O uso de nutracêuticos pode trazer benefícios à saúde, contudo deve ser avaliado o uso caso a caso. Além disso, há a necessidade de uma legislação específica para normatização desses produtos, que alavancaria mais estudos que assegurem a sua eficácia oportunizando um tratamento efetivo e seguro.

Palavras-chave: Ácidos graxos. Flavonoides. Carotenoides. eficácia.

Abstract. Introduction: Nutraceuticals have been highlighted due its health benefits in, preventing and assisting the treatment of diseases. The polyunsaturated fatty acids (PFA), carotenoids, flavonoids and isoflavones are among the most consumed. Objective: The aim was to investigate definitions, regulations, efficacy, types of treatment and adverse reactions of nutraceuticals. Results: PFA have healing and tissue regeneration properties, as well, immunoregulatory, anti-inflammatory properties and in regulating the lipid profile. Beta-carotene prevents cancer, cardiovascular disease, protection of renal function but in smokers it can increase the incidence of lung cancer. Among the flavonoids, quercetin stands for their antioxidant and protective effects on renal, cardiovascular and hepatic functions. However, it should be avoided by hypotensive patients. Isoflavones are useful in symptoms of menopause, including depression. Conclusion: The use of nutraceuticals related to health benefits, however its use should be evaluated in each case. In addition, there is the need for a specific legislation for standardization of these products, which would enhance more studies to ensure their effectiveness and safe treatment.

Key-words: Fatty acids. Flavonoids. Carotenoids. Efficacy.

Resumen. Introducción: Los nutracéuticos vienen destacándose por presentar diversos beneficios a la salud, previniendo y auxiliando en el tratamiento de enfermedades. Los ácidos grasos poli-insaturados (AG), los carotenoides, los flavonoides y las isoflavonas están entre los más consumidos. Objetivo: El objetivo de este trabajo fue investigar definiciones, reglamentaciones, comprobaciones sobre efectividad, tratamiento y reacciones adversas de los nutracéuticos. Resultados: Los AG presentan propiedades cicatrizantes y de regeneración del tejido, inmunorreguladoras, anti-inflamatorias y reguladoras del perfil lipídico. El betacaroteno previene el cáncer, enfermedades cardiovasculares y renais, de entre otras, por otro lado, en fumadores puede aumentar la incidencia de cáncer de pulmón. Entre los flavonoides, la quercetina se destaca por sus efectos antioxidantes, protector de los sistemas renal, cardiovascular y hepático, sin embargo debe ser evitada por pacientes hipotensos. Las isoflavonas son uteis en los síntomas de la menopausa, incluyendo la depresión. Conclusión: El uso de nutracéuticos puede traer beneficios a la salud, no obstante debe ser evaluado el uso caso por caso. Además de eso, hay la necesidad de una legislación específica para la normalización de estos productos, que fomentaría más estudios que aseguren su eficacia, permitiendo un tratamiento efectivo y seguro.

Palabras-clave: Ácidos grasos. Flavonoides. Carotenoides. Efectividad. 
Figura 1. Ilustração do termo Nutracêutico.

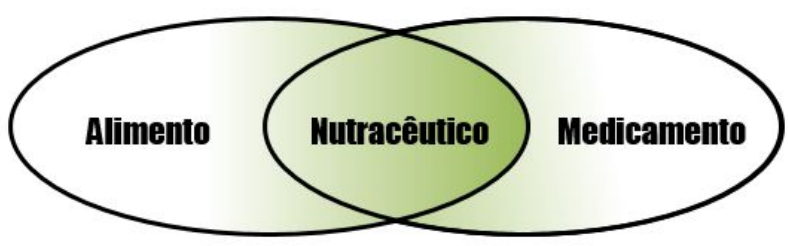

Figura 1. Ilustração do termo Nutracêutico.

\section{Introdução}

Uma alimentação balanceada pode prevenir e tratar muitas doenças, pois há uma estreita relação entre nutrientes e saúde. Hipócrates, o pai da medicina, dizia: "Deixe o alimento ser o medicamento, e o medicamento ser o alimento"(1).

Nutracêuticos possuem diversas definições, entre estas definições, uma das mais aceitas seria a contração dos termos nutrientes + farmacêuticos, ou seja, alimentos, ou parte de alimentos, ou nutrientes, administrados em formas farmacêuticas $(2,3)$.

As classes de nutracêuticos incluem fibras dietéticas, ácidos graxos poli-insaturados, proteínas, peptídeos, aminoácidos ou cetoácidos, minerais, vitaminas e antioxidantes(2).

Desta forma, o objetivo deste artigo foi buscar na literatura estudos que comprovem a eficácia de produtos nutracêuticos, bem como legislação, tratamento, reações adversas, entre outros.

\section{Materiais e Métodos}

Foi realizada uma revisão bibliográfica entre abril e setembro de 2016, na qual foram utilizados como fonte de pesquisa livros, artigos, e resoluções. Para os artigos utilizou-se as bases de dados PubMed e SciELO, utilizando as palavras-chaves: nutracêuticos/ nutraceuticals, ácidos graxos/ fatty acids, flavonoides/ flavonoids, carotenoides/ carotenoids, eficácia/ efficacy. Os critérios de inclusão para os estudos foram a relação destes com evidências científicas relacionadas à eficácia, ao tratamento e às reações adversas e foram excluídos estudos voltados somente ao crescimento do mercado nutracêutico na indústria farmacêutica.

\section{Resultados e discussão \\ Definições e Legislação}

\begin{tabular}{|c|c|}
\hline Autor, Ano & Conceito e Regulamentação \\
\hline $\begin{array}{l}\text { Brasil, Portaria no } \\
398 \text { de } 1999^{(32 .}\end{array}$ & $\begin{array}{l}\text { Define alimento funcional como: "alimento ou ingrediente que, além das funçōes nutricionais básicas, quando } \\
\text { consumido na dieta usual, produz efétos metaboblicos e/ou fisiológicos benéficos à saúde, devendo ser seguro } \\
\text { para o consumo, sem supervisão". }\end{array}$ \\
\hline $\begin{array}{l}\text { Brasil, Resolução } \\
\text { RES n } n^{\circ} 16 \text { de } \\
1999^{(4)} \text {. }\end{array}$ & 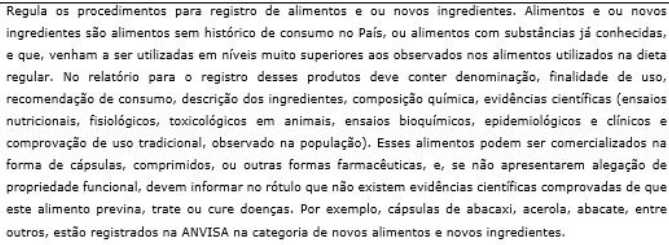 \\
\hline $\begin{array}{l}\text { Brasil, Resoluç̧̋̃o } \\
\text { RES no } 18 \text { de } \\
1999^{(s)} \text {. }\end{array}$ & $\begin{array}{l}\text { Descreve as diretrizes para as alegasóes de propriedades funcionais e ou de saúde de alimentos e ingredientes } \\
\text { para consummo humano, veiculadas nos rótulos de produtos elaborados, embalados e comercializados. }\end{array}$ \\
\hline 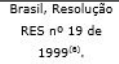 & $\begin{array}{l}\text { Regulamento Técnico de Procedimentos para Registro de Alimentos com Alegação de Propriedades Funcionais } \\
\text { e ou de Saúde em sua Rotulagem. }\end{array}$ \\
\hline $\begin{array}{l}\text { Moraes e Collar } \\
2006^{(2)} \text {. }\end{array}$ & Alimentos funcionais devem estar na forma de alimento e produzem benefícios especificos à saúde. \\
\hline $\begin{array}{l}\text { Brasil, Resolução } \\
\text { RDC no } 22 \text { de } \\
2010^{(n)} \text {. }\end{array}$ & $\begin{array}{l}\text { Aprova o Regulamento Técnico que estabelece as categorias de alimentos e embalagens isentos de registro } \\
\text { sanitário e as categorias de alimentos e embalagens com obrigatoriedade de registro sanitário. Dentre os } \\
\text { alimentos isentos de registro encontram-se: açúcares, chocolates, café, cevada, chá, erva-mate, suplementos } \\
\text { vitaminicos, entre outros. }\end{array}$ \\
\hline $\begin{array}{l}\text { Costa e Rosa, } \\
2010^{(x)}\end{array}$ & $\begin{array}{l}\text { O conceito de alimentos funcionais no Japão recebeu a designą̧ăo de foods for specified health use (FOSHU), } \\
\text { referindo-se aos alimentos usados como parte da dieta que demonstram beneficios fisiológicos e reduzem o } \\
\text { risco de doenças crônicas, além das funç̧oses básicas nutricionais. }\end{array}$ \\
\hline Brasil, $2016^{(\bullet)}$ & 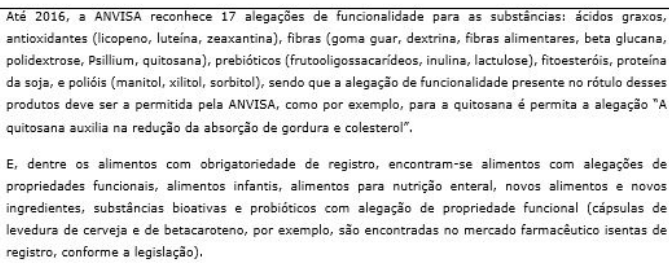 \\
\hline
\end{tabular}

Quadro 1: Conceitos e regulamentação de alimentos funcionais descritos na literatura e nas legislações, com enfoque na legislação brasileira.

Não há uma definição universal para os nutracêuticos, e diversos são os conceitos apresentados por pesquisadores e instituições nacionais e internacionais. É comum considerar os alimentos funcionais e os nutracêuticos como sinônimos(2). As definições de alimentos funcionais e nutracêuticos, encontram-se compilados nos quadros 1 e 2, respectivamente.

Há a necessidade de uma legislação que viabilize adequadamente os nutracêuticos, visto que há um crescente número de publicações sobre a classe(15).

No entanto, os órgãos regulatórios de diferentes países não reconhecem o gênero nutracêutico, enquadrando estes compostos ativos de diferentes formas. Além disso, o cenário de regulamentação sempre esteve sob forte influência do mercado de alimentos funcionais e correlatos(16).

De uma forma geral, considerando todos os conceitos de nutracêuticos descritos, percebe-se uma tendência na padronização do conceito, como alimentos ou parte deles disponibilizados na forma de formas farmacêuticas, em doses maiores das encontradas em alimentos. Nesse contexto, discordamos de incluir dentro da definição de nutracêuticos, alimentos cereais, sopas e bebidas, entre outros. No 


\begin{tabular}{|c|c|}
\hline Autor, Ano & Conceito e Regulamentação \\
\hline $\begin{array}{l}\text { National Institutes of } \\
\text { Health (NIH). U. S. } \\
\text { Department of Health } \\
\text { \& Human Services, } \\
\quad 1994^{(10)} \text {. }\end{array}$ & $\begin{array}{l}\text { Nos Estados Unidos, os nutracêuticos são enquadrados, parcialmente, como um } \\
\text { suplemento dietético. Definem como um alimento, na forma não convencional, capaz } \\
\text { de suplementar a dieta, através do aumento total da ingestão diária deste } \\
\text { componente. O Food and Drug Administration (FDA), através do Dietary Supplement } \\
\text { Health and Education Act (DSHEA), confere uma nova abordagem regulatória para a } \\
\text { segurança e rotulagem de suplementos dietéticos. Segundo o DSHEA, a empresa é } \\
\text { responsável por garantir a segurança do suplemento que fabrica ou distribui; assim, } \\
\text { os suplementos dietéticos não precisam ser submetidos à aprovação do FDA antes de } \\
\text { serem comercializados, exceto no caso de novos compostos. }\end{array}$ \\
\hline $\begin{array}{l}\text { Brasil, Portaria no } 32 \\
\text { de } 1998^{(11)}\end{array}$ & $\begin{array}{l}\text { Regulamento Técnico espećifico para suplementos de vitaminas e minerais, e o } \\
\text { registro dessas substâncias segue o mesmo procedimento que os alimentos. }\end{array}$ \\
\hline Zeisel, 1999(12). & $\begin{array}{l}\text { Define nutracêuticos como: suplementos alimentares que contêm a forma } \\
\text { concentrada de um composto bioativo de alimento, separados da matriz alimentar, } \\
\text { utilizados com a finalidade de melhorar a saúde, em doses que excedem aquelas } \\
\text { obtidas de alimentos. }\end{array}$ \\
\hline $\begin{array}{l}\text { Brasil, Resolução } \\
\text { RDC nº } 2 \text { de } 2002^{(13)} \text {. }\end{array}$ & $\begin{array}{l}\text { Diretrizes a serem adotadas para a avaliação de segurança, registro e comercialização } \\
\text { de substâncias bioativas e probióticos isolados com alegação de propriedades } \\
\text { funcional e, ou, de saúde, apresentadas nas formas sólida, semi-sólida ou líquida, tais } \\
\text { como cápsulas, comprimidos, pós, granulados, pastilhas, suspensões e soluções. A } \\
\text { substância bioativa deve estar presente em fontes alimentares, e pode ser de origem } \\
\text { natural ou sintética. Classificados em: carotenoides, fitoesteróis, flavonoides, } \\
\text { fosfolipidios, organossulfurados, polifenóis e probióticos. Uma vez aprovadas, as } \\
\text { alegações propostas pelo fabricante são obrigatórias. Entretanto, qualquer informação } \\
\text { sobre as propriedades do produto, veiculada por meios de comunicação, não pode ser } \\
\text { diferente daquela aprovada para a rotulagem. }\end{array}$ \\
\hline Kalra, $2003^{(14)}$. & $\begin{array}{l}\text { O fundador e presidente da Foundation for Innovation in Medicine, o médico Stephen } \\
\text { Defelice define nutracêutico como um alimento ou parte de um alimento que } \\
\text { proporciona beneficios para a saúde, incluindo a prevenção ou tratamento de doença. } \\
\text { Tais produtos podem variar desde nutrientes isolados, suplementos dietéticos a } \\
\text { alimentos geneticamente modificados, produtos herbais e alimentos processados tais } \\
\text { como cereais, sopas e bebidas. }\end{array}$ \\
\hline $\begin{array}{l}\text { Moraes e Colla, } \\
2006^{(2)} \text {. }\end{array}$ & $\begin{array}{l}\text { Nutracêuticos são alimentos ou parte dos alimentos, na forma de medicamentos, que } \\
\text { apresentam beneficios à saúde, incluindo a prevenção e/ou tratamento de doenças. }\end{array}$ \\
\hline
\end{tabular}

Quadro 2: Conceitos e regulamentação de nutracêuticos descritos na literatura e nas legislações, com enfoque na legislação brasileira.

Brasil, os nutracêuticos, comercializados na forma de cápsulas, por exemplo, devem seguir basicamente a RES n ${ }^{\circ} 16$ de 1999(4) ou a RDC $n^{\circ} 2$ de 2002(13). A primeira, para alimentos processados e encapsulados, e a segunda, quando se usa um ingrediente isolado, por exemplo, para registrar o alimento tomate na forma de cápsulas, segue a (4), e se for registrar o licopeno, bioativo do extrato de tomate, segue a (13).

Recentemente, no Brasil, em julho de 2018, a ANVISA publicou uma notícia para regulamentar os suplementos por meio de consultas públicas e, em breve, serão publicadas as novas legislações acerca do assunto $(17,18)$.

Nutracêuticos comumente utilizados e seus benefícios

Ácidos graxos poli-insaturados (Polyunsaturated fatty acids: PUFAs): Ômega 3 e Ômega 6

Os PUFAS ômega-3 e ômega-6 são obtidos por meio da dieta ou produzidos pelo organismo a partir dos ácidos linoleico e alfa-linolênico. São necessários para manter sob condições normais, as membranas celulares, as funções cerebrais e a transmissão de impulsos nervosos. Esses ácidos graxos também participam da transferência do oxigênio atmosférico para o plasma sanguíneo, da síntese da hemoglobina e da divisão celular(19).

As principais fontes alimentares do ácido linoleico são: os óleos de soja, girassol e milho, e do ácido alfa-linolênico são: os óleos de linhaça, canola e peixes(20).

Entre as principais funções estão o depósito de energia e a conformação das membranas celulares, sendo também precursores de substâncias, como as prostaglandinas, tromboxanos e leucotrienos. Previnem e tratam doenças cardiovasculares, hipertensão, inflamações em geral, asma, artrite, psoríase e vários tipos de câncer(21). A suplementação com ômega-3 pode prevenir riscos cardiovasculares(22).

A suplementação no pré-operatório com ômega-3 favoreceu a cicatrização de anastomoses colônicas em ratos Wistar, adultos machos pois, aumentou a deposição de colágeno nas anastomoses de cólon no $5^{\circ}$ dia do pósoperatório(23).

Em outro estudo, a suplementação com ômega-3 (2g/dia) em indivíduos submetidos a reconstrução do ligamento cruzado do joelho impediu a ação dos radicais livres nas estruturas periarticulares e o agravamento das lesões no joelho(24).

EPA (ácido eicosapentaenoico) e DHA (ácido docosaexaenoico) são os principais bioativos do ômega-3. O ALA (ácido alfa linolênico), precursor de EPA e DHA é geralmente menos efetivo em induzir efeitos biológicos, em parte pela sua ineficiente conversão em EPA e DHA em huma$\operatorname{nos}(25)$.

Em ensaios clínicos, EPA e DHA preveniram doenças periodontais e efeitos antibacterianos frente a patógenos periodontais(26).

Em ratos Wistar, machos e fêmeas, com oito semanas de idade, dietas ricas em ômega-3 melhoram o perfil hepático, levando ao menor risco de desenvolver síndrome metabólica relacionada ao perfil lipídico(27).

Ensaios clínicos de EPA entre grupos de alto risco no Japão em que o consumo de ácidos graxos ômega-3 é maior do que em outros países mostraram progressão mais lenta de aterosclerose coronariana(28).

O equilíbrio entre ômega- 6 e ômega-3 é um fator determinante para reduzir riscos de problemas cardíacos na prevenção primária e secundária de doença coronariana(29).

A suplementação com ômega-3 altera os indicadores bioquímicos do metabolismo lipídico, reduzindo as lipoproteinas ricas em colesterol plasmático, prevenindo doenças cardiovasculares, segundo estudo em nadadores(30).

Recente meta-análise e meta-regressão de controles aleatórios de ensaios clínicos randomizados sugerem que a 
suplementação com PUFA ômega-3 podem modificar favoravelmente biomarcadores cardiometabólicos no diabetes tipo 2 e, melhora do perfil lipídico e de marcadores de inflamação sem efeitos adversos no LDL ou HbA1c(31).

Em estudo clínico, com pacientes hipercolesterolêmicos não tratados farmacologicamente, comparou-se dois tratamentos administrados por 8 semanas. Um dos grupos usou uma combinação de arroz vermelho fermentado (Dif1Stat ${ }^{\circledR}$, incluindo $5 \mathrm{mg}$ de monacolina K) e $610 \mathrm{mg}$ de PUFAs e, o outro fitoesteróis $1600 \mathrm{mg} / \mathrm{dia}$. Ambos os grupos apresentaram diminuição significativa nos níveis de colesterol total, colesterol de lipoproteína de baixa densidade, triglicérides e não-HDL-C, porém no grupo contendo arroz+PUFAs a diminuição foi maior. A curto prazo esse tratamento mostrou-se bem tolerado e eficaz na redução de lipídios plasmáticos(32).

Um estudo com ratos com periodondite apical (PA) concluiu que a presença de múltiplos focos de PA aumenta os níveis de triglicerídeos e a suplementação com ômega-3 pode reduzir estes níveis, bem como a área de reabsorção óssea dos tecidos periapicais(33). Em cabras, também foi observada uma melhora significativa nos níveis de triglicerídeos e colesterol total no grupo suplementado com ômega-3(34).

Neste contexto, os estudos mostram que PUFAs auxiliam no processo de cicatrização e regeneração tecidual, devido a suas propriedades imunorreguladoras, antioncogênicas, anti-inflamatórias e, especialmente reguladoras do perfil lipídico.

\section{VITAMINAS E ANTIOXIDANTES}

\section{Betacaroteno}

O betacaroteno é o pigmento natural mais abundante dos carotenoides. É encontrado especialmente em vegetais e frutas de cor amarelo alaranjada e em vegetais folhosos de cor verde-escura $(35,36)$.

É o único carotenoide que apresenta dois radicais ionona, que ao romper-se forma duas moléculas de próvitamina $A(36)$, ou seja, o betacatoteno é um precursor da produção de vitamina $A$.

A principal atividade antioxidante dos carotenoides se deve a capacidade de desativar o oxigênio singleto e neutralizar radicais peroxil, eliminando radicais de oxigênio e, reduzindo a oxidação do DNA e de lipídios que está associada a doenças degenerativas, como câncer e doenças cardíacas. Dessa forma, o betacaroteno age sobre as células imunocompetentes, aumentando os linfócitos $\mathrm{T}$ e as células natural killers(37).

O betacaroteno é capaz de proteger contra danos oxidativos do tecido renal de ratos com toxicidade renal induzida por tioacetamida(38).
O potencial quimiopreventivo do betacaroteno contra o câncer, tem sido evidenciado em estudos epidemiológicos observacionais e experimentais(37).

O betacaroteno tem sido usado para tratar lesões préneoplásicas, como a leucoplaquia oral. Em estudos realizados com tabagistas americanos, observou-se que a suplementação com betacaroteno (30mg/dia, por 3-6 meses) causou remissão parcial ou completa de lesões pré-neoplásicas da cavidade oral, em mais de $70 \%$ dos casos(39).

Por outro lado, a suplementação com betacaroteno em indivíduos expostos ao fumo intenso pode aumentar a incidência de câncer de pulmão, que estaria relacionado com a oxidação do betacaroteno pelos compostos presentes na fumaça do cigarro(40).

Estudos epidemiológicos encontraram associação benéfica entre a ingestão de vitaminas antioxidantes e o risco para câncer cervical, onde o betacaroteno demonstrou capacidade protetora principalmente nos estágios iniciais da carcinogênese cervical. Entre as funções do carotenoide, está a capacidade de inibir a oxidação de compostos pelos peróxidos, impedindo o crescimento de células malignas no epitélio escamoso do colo uterino com consequente inibição do desenvolvimento do HPV e evolução das lesões displásicas. Um estudo experimental randomizado realizado com 69 mulheres, em que 39 receberam betacaroteno e 30 receberam placebo durante nove meses, evidenciou que concentrações baixas de carotenoides no sangue estão associadas a um risco aumentado de neoplasia intraepitelial cervical (NIC). No grupo de tratamento, a regressão da NIC ocorreu em $33 \%$ e, no grupo placebo a regressão foi de $20 \%(41)$.

Outro estudo com índias americanas do Sudoeste, entre 18 e 45 anos, onde 81 mulheres apresentavam NIC e 160 mulheres apresentavam epitélio cervical normal, foi demonstrado que a ingestão aumentada de carotenoides, vitamina A e retinol está associado com a diminuição de $60 \%$ do risco de desenvolvimento de lesões precursoras e câncer cervical. Os carotenoides séricos têm propriedades antioxidantes e podem impedir a ação de radicais livres que induzem danos no DNA e consequentemente a carcinogênese cervical(42).

O betacaroteno também reduziu significativamente a pressão arterial de ratos espontaneamente hipertensos, sem efeitos tóxicos ou alterações dos parâmetros biológicos(43).

A vitamina A participa também do processo de formação da pele, unhas e cabelo, bem como atua na queratinização e estimula a microcirculação cutânea, com potencial atividade antienvelhecimento. Atualmente, foram obtidas formas farmacêuticas mais estáveis e ativas dessa substância, de maneira a aumentar sua ação(37).

Os carotenoides, usados individualmente ou combinados com outros carotenoides ou vitaminas, podem proteger os tecidos expostos à luz, sendo usados combinados com filtros solares para a prevenção de queimaduras solares. Utilizados via oral têm uma atividade muito menor quando 
comparados com o uso tópico. Na pele, os carotenoides estão presentes na corrente sanguínea funcionando como um meio de armazenamento estável, prevenindo o envelhecimento prematuro da pele e certos tipos de câncer de pele(44).

É importante ficar atento aos níveis de segurança do seu consumo de modo a evitar uma hipervitaminose A aguda. Os sintomas são dores de cabeça, dores ósseas e articulares, náuseas e pele seca. O problema mais grave associado com a hipervitaminose A é a teratogenicidade(44).

Entre os diversos benefícios da vitamina A, é evidente que o principal é sua atividade antioxidante e, consequentemente redução de radicais livres e de câncer. Tem um papel importante na proteção da pele frente a queimaduras solares e, potencial antienvelhecimento.

\section{Quercetinas}

Flavonoides são compostos polifenólicos biossintetizados a partir da via dos fenilpropanoides e do acetato, precursores de vários grupos de substâncias como aminoácidos alifáticos, terpenoides, AG, dentre outros. Os flavonoides representam um dos grupos fenólicos mais importantes e diversificados entre os produtos de origem natural(45).

A quercetina é o principal flavonoide presente na dieta humana. É encontrada em grandes concentrações em alguns alimentos como cebolas, maçãs, vinho tinto, brócolis e chás(46). Várias propriedades terapêuticas dos flavonoides, principalmente da quercetina, vêm se destacando devido ao potencial antioxidante, anticarcinogênico e seus efeitos protetores aos sistemas renal, cardiovascular e hepático(47).

As propriedades antioxidantes da quercetina estão relacionadas com a sua estrutura química. Atua como um protetor contra as espécies reativas de oxigênio, neutralizando os radicais livres e ânions superóxido. O efeito antioxidante também está relacionado a sua capacidade de inibir enzimas, tais como oxidase de xantina, da NADPH-oxidase e lipoxigenase, prevenindo a morte celular(2).

A quercetina apresentou efeito protetor nas alterações histomorfométricas do rim de fetos de ratos tratados com ácido retinoide(48).

A atividade anticarcinogênica dos flavonoides, em especial da quercetina, se dá pela inibição do GLUT-1 (Glucose Transporter 1), que possui um papel direto no transporte de glucose e, na oncogênese, como no câncer hepatocelular (CHC). Em uma linhagem celular humana de $\mathrm{CHC}$, verificou-se que a quercetina é um inibidor competitivo do GLUT-1, afetando deste modo a captação de glucose e seus análogos, inibindo a proliferação da linha celular, apresentando efeito anticarcinogênico, sem efeito citotóxico(49).

A quercetina apresenta também efeitos ergogênicos. No entanto, para isso a suplementação é fundamental, pois, a quantidade mínima necessária para que a quercetina exerça seus efeitos ergogênicos é de $1 \mathrm{~g}$ /dia, não sendo possível ingerir somente pela dieta. A suplementação de quercetina melhorou a capacidade de resistência na prática de exercí$\operatorname{cios}(50)$.

Um estudo experimental avaliou os efeitos da luteolina e da quercetina presentes em folhas de Passiflora subpeltata na hepatotoxicidade induzida por paracetamol em ratos Wistar machos. Os animais tratados com extratos das folhas de Passiflora subpeltata, tiveram um aumento de enzimas antioxidantes o que levou a diminuição da peroxidação de lipídios, controlando os danos hepáticos(51).

A quercetina $(10 \mathrm{mg} / \mathrm{ml})$ exibiu atividade antiviral in vitro contra o parvovírus canino, que pode estar relacionada à inativação direta do vírus por meio da ligação a estruturas virais essenciais para a infecção, sugerindo o uso desse flavonoide como candidato a agente terapêutico no controle da parvovirose canina $(52)$.

A quercetina demonstrou efeitos terapêuticos antiapoptóticos contra morte celular por apoptose induzida por lesão na retina de ratos por meio da elevação da pressão intraocular, apresentando benefícios quando o mecanismo fisiopatológico envolve lesão(53).

A suplementação com quercetina em doses de 500 a $1500 \mathrm{mg}$ é considerada segura(54). No entanto, em algumas apresentações comercias, recomenda-se dose de até $2000 \mathrm{mg} /$ dia, mais do que investigado na clínica, na qual não existem dados que garantam sua segurança(55).

No que diz respeito à contraindicação, a quercetina deve ser evitada por pacientes hipotensos e com transtornos de coagulação, pois os flavonoides têm efeito vasodilatador. Também, não deve ser utilizada concomitante aos antibióticos da família das quinolonas, pois é possível que iniba a ação do fármaco(55).

Em estudos in vitro observou-se atividade mutagênica da quercetina em diferentes cepas de Salmonolla typhimurium e Bacillus subtilis. No entanto, em estudos realizados in vivo não se tem observado toxicidade do flavonoide. Desta forma, a quercetina via oral não produz efeitos adversos que comprometam a saúde(56).

Recente trabalho sugere efeito anti-inflamatório da quercetina na região hipocampal CA1 em um modelo para a doença de Alzheimer em camundongos transgênicos, reduzindo a agregação de placas -amiloide(57).

Dentre os principais efeitos da quercetina, estão os antioxidantes, anti-câncer, anti-inflamatórios, e vasodilatador. E, é necessária suplementação oral, além da dieta, quando se quer efeitos diretos do flavonoide.

Isoflavonas de Soja 
As isoflavonas são compostos pertencentes ao grupo dos flavonoides, que se caracterizam por apresentar estrutura polifenólica. Podem agir como antioxidantes, antiinflamatórios, antimicrobianos entre outras atividades biológicas, tornando os produtos que as contém em alimento funcional ou nutracêutico(58). Estão presentes principalmente em produtos à base de soja, mas também em outros grãos como na ervilha verde, lentilha, feijão e seus derivados e em legumes(59).

As isoflavonas podem ser recomendadas como tratamento de primeira escolha contra afrontamentos da menopausa. O uso de $150 \mathrm{mg}$ de isoflavona por dia por pelo menos três anos pode ainda ter efeitos preventivos contra o câncer de mama e também, pode ser usado concomitante ao tratamento com anastrozol ou tamoxifeno(60).

Um ensaio clínico com mulheres na menopausa evidenciou que uma bebida a base de soja, ingerida durante 12 semanas, reduziu os sintomas climatéricos, principalmente os mais incômodos, como as ondas de calor(61).

Um estudo de intervenção dietética de 8 semanas em 57 mulheres pós-menopáusicas saudáveis investigou a suplementação alimentar de soja (50 mg de isoflavonas ou $15 \mathrm{~g}$ de proteína de soja) e demonstrou efeitos benéficos na redução dos níveis séricos de gordura(62). Outro estudo prospectivo com 50 mulheres na menopausa confirmou o potencial de redução de taxas hipercolesterolêmicas das isoflavonas(63).

As isoflavonas auxiliam na redução dos sintomas depressivos da menopausa. Em um estudo placebo-controlado, randomizado e duplo-cego com 87 pacientes climatéricas foi observado efeito positivo no tratamento dos sintomas do climatério, podendo beneficiar pacientes que decidem não usar estrógeno ou que apresentam efeitos colaterais pelo uso desses hormônios, considerando que não houve relatos de efeitos adversos durante o uso das isoflavonas(64).

A administração de isoflavona durantes doze semanas em um estudo randomizado, duplo cego, controlado por placebo em 70 mulheres com Síndrome do Ovário Policístico demonstrou uma melhora significativa nos marcadores de resistência à insulina, no estado hormonal, nos níveis de triglicerídeos e biomarcadores de estresse oxidativo(65).

O consumo de soja tem sido associado à redução de doenças cardiovasculares, especialmente da aterosclerose em modelos animais. Além disso, evidências epidemiológicas sugerem que populações que consomem dietas ricas em soja e seus produtos apresentam uma menor taxa de mortalidade por doenças coronarianas(66).

Recente estudo avaliou os efeitos neuroprotetores de isoflavonas de soja na amnésia induzida por escopolamina em camundongos e observou efeito neuroprotetor sobre as disfunções cognitivas, sugerindo que as isoflavonas podem ser candidatas para o tratamento de doenças neurodegenerativas, como a doença de Alzheimer(67).

O consumo de soja permite redução do colesterol total, do LDL-colesterol e dos triglicerídeos. Supõe-se que os fitoestrógenos estimulam a secreção de sais biliares e em consequência, aumentam a taxa de excreção do colesterol(68).

Foi realizado um estudo multicêntrico, randomizado e duplo cego entre maio de 2010 e agosto de 2012 para investigar o efeito de um suplemento de isoflavona de soja sobre a função pulmonar em pacientes com asma mal controlada. Em comparação com o placebo, o uso da isoflavona não resultou em melhoria da função pulmonar. Sugerindo que este suplemento não deve ser utilizado por pacientes asmáticos(69).

Em contrapartida, a toxicidade puberal de uma alimentação rica em leite de soja (suplementada ou não com glifosato, doses de 50 e $100 \mathrm{mg} / \mathrm{kg}$ ) em ratos machos foi avaliada. A desregulação endócrina foi observada através da diminuição dos níveis de testosterona, diminuição do número de células de Sertoli e aumento na porcentagem de células de Sertoli e Leydig degeneradas em animais recebendo leite de soja suplementado com glifosato (ambas as doses) e em animais tratados somente com leite de soja. O leite de soja apresenta efeito disruptor endócrino, devido as diminuições dos níveis de testosterona(70).

Os principais estudos das isoflavonas concentram-se nos benefícios obtidos em mulheres na menopausa. Neste sentido, as isoflavonas podem diminuir os efeitos do climatério, reduzir a depressão associada ao período, bem como, reduzir níveis lipídicos. Devido ao efeito no sistema reprodutor de ratos machos, não se recomenda o uso em pacientes do sexo masculino.

\section{Considerações finais}

Os nutracêuticos promovem benefícios à saúde, e seu uso está em constante crescimento, sendo um mercado altamente promissor. Além dos benefícios, alguns efeitos colaterais já estão descritos, por isso, a necessidade de orientação de profissionais da saúde capacitados para avaliar as condições de saúde do indivíduo e após prescrever o uso de nutracêuticos.

Os nutracêuticos podem ser utilizados como coadjuvantes no tratamento de patologias ou de forma preventiva, entretanto há ainda, uma grande lacuna de desenvolvimento científico na área.

Nesse sentido, percebe-se que há a necessidade de uma legislação específica que defina oficialmente o termo nutracêutico e regulamente o processo de produção e comercialização desses produtos, garantindo desta forma a qualidade e segurança do produto final, além de alavancar as pesquisas na área.

\section{Referências}

1. Silva IMC, Sá EQC. Alimentos funcionais: um enfoque gerontológico. Rev Bras Clín Méd 2012;10(1):24-28. 
2. Moraes FP, Colla LM. Alimentos funcionais e nutracêuticos: definições, legislação e benefícios à saúde. Rev Eletr Farm 2006;3(2):109-122.

3. Brasil. Agência Nacional de Vigilância Sanitária. Portaria ${ }^{\circ} 398$, de 30 de abril de 1999. Regulamento Técnico que Estabelece as Diretrizes Básicas para Análise e Comprovação de Propriedades Funcionais e ou de Saúde Alegadas em Rotulagem de Alimentos. Diário Oficial da União 1999; 03 maio.

4. Brasil. Agência Nacional de Vigilância Sanitária. Resolução RES 16, de 30 de abril de 1999. Aprova o Regulamento Técnico de Procedimentos para registro de Alimentos e ou Novos Ingredientes. Diário Oficial da União 1999; 03 dez.

5. Brasil. Agência Nacional de Vigilância Sanitária. Resolução RES 18, de 30 de abril de 1999. Aprova o Regulamento Técnico que estabelece as diretrizes básicas para análise e comprovação de propriedades funcionais e ou de saúde alegadas em rotulagem de alimentos. Diário Oficial da União 1999; 03 maio.

6. Brasil. Agência Nacional de Vigilância Sanitária. Resolução RES 19, de 30 de abril de 1999. Aprova o Regulamento Técnico de procedimentos para registro de alimento com alegação de propriedades funcionais e ou de saúde em sua rotulagem. Diário Oficial da União 1999; 03 maio.

7. Brasil. Agência Nacional de Vigilância Sanitária. Resolução RDC 27, de 06 de agosto de 2010. Aprova o Regulamento Técnico que estabelece as categorias de alimentos e embalagens isentos de registro sanitário e as categorias de alimentos e embalagens com obrigatoriedade de registro sanitário. Diário Oficial da União 2010; 09 ago.

8. Costa NMB, Rosa COB. Alimentos Funcionais Componentes Bioativos e Efeitos Fisiológicos. Rio de Janeiro: Editora Rubio; 2010.

9. Brasil. Agência Nacional de Vigilância Sanitária. Alimentos Com Alegações de Propriedades Funcionais e ou de Saúde. 2016. [acesso em 2017 jan. 12]. Disponível em: http://portal.anvisa.gov.br/alimentos/alegacoes.

10. National Institutes of Health (NIH). UNITED STATES OF AMERICA. U. S. Department of Health Human Services. Office of Dietary Supplements. Dietary supplement health and education act of 1994. Public law 103-417. 109rd Congress. [acesso em 2016 jun. 6]. Disponível em: https://ods.od.nih.gov/About/DSHEA ${ }_{W}$ ording.aspx.

11. Brasil. Agência Nacional de Vigilância Sanitária. Portaria $\mathrm{n}^{\circ}$ 32, de 13 de janeiro de 1998. Aprova o Regulamento Técnico para Suplementos Vitamínicos e ou de Minerais. Diário Oficial da União 1998; 15 jan.

12. Zeisel SH. Regulation of "Nutraceuticals."Science 1999;285:1853-1855. DOI: 10.1126/science.285.5435.1853.

13. Brasil. Agência Nacional de Vigilância Sanitária. Resolução RDC 2, de 07 de janeiro de 2002. Aprova o Regulamento Técnico de Substâncias Bioativas e Probióticos Isolados com Alegação de Propriedade Funcional e ou de Saúde. Diário Oficial da União 2002; 09 jan.

14. Kalra EK. Nutraceutical - Definition and Introduction. AAPS Pharm Sci 2003;5(3):E25. DOI: http://dx.doi.org/10.1208/ps050325.

15. Coppens P, Silva MF, Pettman S. European regulations on nutraceuticals, dietary supplements and functional foods: a framework based on safety. Toxicology 2006;221:59-74. DOI: http://dx.doi. org/10.1016/j.tox.2005.12.022.

16. Hasler CM. Regulation of functional foods and nutraceuticals: a global perspective. Iowa: Blackwell Publishing; 2005.

17. Brasil. Agência Nacional de Vigilância Sanitária. Suplementos: Anvisa quer regulação específica. 2018. [acesso em 2018 ago. 10]. Disponível em: http://portal.anvisa.gov. br/noticias?p_p_id=101_INSTANCE_FXrpx9qY7FbU\&p_p_col_id=column2\&p_p _col_count $=2 \&$ 101_INSTANCE_FXrpx9qY7FbU_grou$\overline{\mathrm{pId}}=219201 \&$ 101_INSTANCE_FXrpx9qY7FbU_urlTitle =anvisa-quer-regulacao- especifica-para-suplementosalimentares \&_101_INSTANCE_FXrpx9qY7FbU_struts 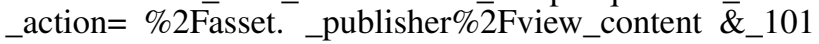 _INSTANCE_FXrpx9qY7FbU_type=content

18. Brasil. Agência Nacional de Vigilância Sanitária. Suplementos alimentares ganham regulamentação inédita. 2018. [acesso em 2018 ago. 10]. Disponível em: http://portal.anvisa.gov.br/rss// asset_publisher/Zk 4q6UQCj9Pn/content/id/4656534

19. Martin CA, Almeida VV, Ruiz MR, Visentainer JEL, Matshushita M, Souza NE et al. Ácidos graxos poliinsaturados ômega-3 e ômega-6: importância e ocorrência em alimentos. Rev Nutr 2006;19(6):761-770. DOI: http://dx.doi.org/10.1590/S1415-52732006000600011.

20. Mesquita TR, Souza AA, Constantino E, Pelógia NCC, Posso IP, Pires OC. Efeito anti-inflamatório da suplementação dietética com ácidos graxos ômega3, em ratos. Rev dor 2011;12(4):337-341. DOI: http://dx.doi.org/10.1590/S1806-00132011000400010.

21. Martin CA, Almeida VV, Ruiz MR, Visentainer JEL, Matshushita M, Souza NE, Visentainer JV. Ácidos graxos poliinsaturados ômega-3 e ômega-6: importância e ocorrência em alimentos. Rev Nutr 2006;19(6):761-770. DOI: http://dx.doi.org/10.1590/S1415-52732006000600011.

22. Bansal S, Buring JE, Rifai N, Mora S, Sacks FM, Ridker PM Fasting compared with nonfasting triglycerides and risk of cardiovascular events in women. JAMA 2007;298:309-316. DOI: http://dx.doi.org/ 10.1001/jama.298.3.309.

23. Castilho TJC, Campos AC, Mello EVSL. Effect of ômega-3 fatty acid in the healing process of colonic anastomosis in rats. Aq Bras Cir Dig 2015;28(4):258-261. DOI: http://dx.doi.org/10.1590/S0102-6720201500040010.

24. Vidmar MF, Siqueira LO, Brito VB, Martins CAQ, Pimentel GL, Almeida CR, Rosa LHT, Silva MF. Suplementação com ômega-3 pós-reconstrução do ligamento cruzado anterior. Rev Bras Med Esporte 2016;22(2):131-137. DOI: http://dx.doi.org/10.1590/1517-869220162202152503.

25. Torrejon C, Jung UJ, Deckelbaum RJ. Fatty Acids and Cardiovascular Disease: Actions and Molecular Mechanisms. Prostaglandins Leukot Essent Fatty Acids 2008;77(5):319-326. DOI: http://dx.doi. org/10.1016/j.plefa.2007.10.014.

26. Sun M, Zhou Z, Dong J, Zhang J, Xia Y, Shu R. Antibacterial and antibiofilm activities of docosahexaenoic 1 acid (DHA) and eicosapentaenoic acid (EPA) against periodontopathic bacteria. Microb Pathog 2016;99(16):196-203. DOI: $10.1016 /$ j.micpath.2016.08.025. 
27. Halfen S, Jacometo CB, Mattei P, Fenstenseifer SR, Pfeifer LFM, Pino FABD, Santos MAZS, Pereira CMP, Schmitt E, Corrêa MN. Diets Rich in Polyunsaturated Fatty Acids With Different Omega-6/Omega-3 Ratio Decrease Liver Content of Saturated Fatty Acids Across Generations of Wistar Rats. Braz Arch Biol Technol 2015;59:1-9. DOI: http://dx.doi.org/10.1590/1678-4324-2016150549.

28. Sekikawa A, Mahajan H, Kadowaki S, Hisamatsu T, Miyagawa N, Fujiyoshi A et al. Association of blood levels of marine omega- 3 fatty acids with coronary calcification and calcium density in Japanese men. Eur J Clin Nutr 2018 Jul 26. DOI: 10.1038/s41430-018-0242-7.

29. Simopoulos AP. The Importance of the Omega6/Omega-3 Fatty Acid Ratio in Cardiovascular Disease and Other Chronic Diseases. Exp Biol Med (Maywood) 2008;233(6):674-688. DOI: 10.3181/0711-MR-311.

30. Andrade PMM, Ribeiro BG, Campo MGT. Omega 3 fatty acids-supplementation to competition athletes: impact on the biochemical indicators related to the lipid metabolism. Rev Bras Med Esporte 2006;12(6):303-307. DOI: http://dx.doi.org/10.1590/S1517-86922006000600008.

31. O’Mahoney LL, Matu J, Price OJ, Birch KM, Ajjan RA, Farrar D et al. Omega-3 polyunsaturated fatty acids favourably modulate cardiometabolic biomarkers in ype 2 diabetes: a meta-analysis and meta-regression of randomized controlled trials. Cardiovasc Diabetol 2018;17(1):98. DOI: 10.1186/s12933-018-0740-x.

32. Cicero AF, Derosa G, Pisciotta L, Barbagallo C, Sisa-Pufacol Study Grup. Testing the Short-Term Efficacy of a Lipid-Lowering Nutraceutical in the Setting of Clinical Practice: A Multicenter Study. J Med Food 2015;18(11):1270-1273. DOI: 10.1089/jmf.2015.0024.

33. Azuma MM, Gomes-Filho JE, Cardoso CBM, Pipa CB, Narciso LG, Bomfim SRM et al. Omega 3 Fatty Acids Reduce the Triglyceride Levels in Rats with Apical Periodontitis. Braz Dent J 2018;29(2):173-178. DOI: http://dx.doi.org/10.1590/0103-6440201801702.

34. Teama FEI, El-Tarabany AA. Physiological and biochemical response to Omega-3 plus as a dietary supplement to growing goats under hot summer conditions. Rev Bras Zootec 2016;45(4):174-180. DOI: http://dx.doi.org/10.1590/S1806-92902016000400005.

35. Naves MMV. Beta-Caroteno e Câncer. Rev Nutr 1998;11(2):99-115. DOI: http://dx.doi.org/10.1590/S141552731998000200001.

36. Ambrósio CLB, Campos FACS, Faro ZP. Carotenoides como alternativa contra a hipovitaminose A. Rev Nutr 2006;19(2):233-243. DOI: http://dx.doi.org/10.1590/S141552732006000200010.

37. Santos MP, Oliveira NRF. Ação das vitaminas antioxidantes na prevenção do envelhecimento cutâneo. Disciplinarum Scientia 2014;15(1):75-89.

38. Fazal Y, Fatima SN, Shahid SM, Mahboob T. Nephroprotective effects of -carotene on ACE gene expression, oxidative stress and antioxidant status in thioacetamide induced renal toxicity in rats. Pak J Pharm Sci 2016;29(4):1139-1144.

39. Garewal HS. Antioxidants in oral cancer prevention. Am J Clin Nutr 1995;62(supl 6):1410S-1416S.

40. Baker DL, Krol ES, Jacobsen N, Liebler DC. Reactions of beta-Carotene with Cigarette Smoke
Oxidants. Identification of Carotenoid Oxidation Products and Evaluation of the Prooxidant/Antioxidant Effect. Chem Res Toxicol 1999;12(6):535-543. DOI: http://dx.doi.org/10.1021/tx980263v.

41. Marshall K. Cervical dysplasia: early intervention. Altern Med Ver 2003;8(2):156-170.

42. Sapaio LC, Almeida CF. Vitaminas Antioxidantes na Prevenção do Câncer do Colo Uterino. Rev Bras Cancerol. 2009;55(3):289-296.

43. Oliveira GS, Figueiredo ASP, Santos RS, Vianna LM. Efeito da suplementação de beta-caroteno na pressão arterial de ratos. Rev Nutr 2007;20(1):39-45. DOI: http://dx.doi.org/10.1590/S1415-52732007000100004.

44. FOOD SUPPLEMENTS EUROPE (FSE). Facts about vitamins, minerals and other food components with health effects. 2013 [acesso em 2016 jul 20]; Disponível em: http://www.foodsupplementseurope.org/sites/0023/uploads /content/publications/facts-about-vitamins-minerals.pdf.

45. Dornas WC, Oliveira TT, Rodrigues-das-Dores RG, Santos AF, Nagem TJ. Flavonóides: potencial terapêutico no estresse oxidativo. Rev Ciênc Farm Básica Apl 2007;28(3):241-249.

46. Panchal SK, Poudyal H, Brown L. Quercetin Ameliorates Cardiovascular, Hepatic, and Metabolic Changes in Diet-Induced Metabolic Syndrome in Rats. J Nutr 2012;142(6):1026-1032. DOI: 10.3945/jn.111.157263.

47. Behling EB, Sendão MC, Francescato HDC, Antunes LMG, Bianchi MLP. Flavonóide Quercetina: Aspectos Gerais e Ações Biológicas. Alim Nutr 2004;15(3):285-292.

48. Khaksary-Mahabady M, Ranjbar R, NajafzadehVarzi H, Mohammadian B, Gohari-Behbahani N. Protective Effect of Quercetin on Histomorphometric Changes in Kidney of Retinoid Acid-Treated Rat Effects. Int J Morphol 2018;36(1):338-344. DOI: http://dx.doi.org/10.4067/S071795022018000100338.

49. Brito AF, Ribeiro M, Abrantes AM, Gonçalves AC, Sarmento-Ribeiro AB, Tralhão JG, Botelho MF. Ácção anticancerígena da Quercetina no Carcinoma Hepatocelular: o papel do GLUT-1. Rev Port Cir 2013;(25):23-30.

50. Kressler J, Millard-Stafford M, Warren GL. Quercetin and Endurance Exercise Capacity: A Systematic Review and Meta-analysis. Med Sci Sports Exerc 2012;44(3):558559. DOI: 10.1249/MSS.0b013e31822495a7.

51. Shanmugama S, Thangarajb P, Lima BS, Chandran R, Araújo AAS, Narain N, Serafini MR, Quintans LJ Jr. Effects of luteolin and quercetin 3-b-D-glucoside identified from Passiflora subpeltata leaves against acetaminophen induced hepatotoxicity in rats. Biomed Pharma 2016;83:1278-1285. DOI: 10.1016/j.biopha.2016.08.044.

52. Carvalho O, Oliveira FS, Saraiva GL, et al. Potencial antiviral da quercetina sobre o parvovirus canino. Arq Bras Med Vet Zootec 2013;65(2):353-358. DOI: http://dx.doi.org/10.1590/S0102-09352013000200008.

53. Arikan S, Ersan I, Karaca T, Kara S, Gencer B, Karaboga I, Hasan Ali T. Quercetin protects the retina by reducing apoptosis due to ischemia-reperfusion injury in a rat model. Arq Bras Oftalmol 2015;78(2):100-104. DOI: 10.5935/0004-2749.20150026.

54. Valério DA, Georgetti SR, Magro DA, Casagrande R, Cunha TM, Vicentini FT, Vieira SM, Fonseca MJ, Ferreira SH, Cunha FQ, Verri WA Jr. Quercetin Reduces 
Inflammatory Pain: Inhibition of Oxidative Stress and Cytokine Production. J Nat Prod 2009;72(11):1975-1979. DOI: $10.1021 / \mathrm{np} 900259 \mathrm{y}$.

55. Vicente-Vicente L, Prieto M, Morales AI. Eficacia y seguridad de la quercetina como complemento alimenticio. Rev Toxicol 2013;30(2):171-181.

56. Harwood M, Daniele-Nikiel B, Borzelleca JF, Flamm GW, Williams GM, Lines TC. A critical review of the data related to the safety of quercetin and lack of evidence of in vivo toxicity, including lack of genotoxic/carcinogenic properties. Food Chem Toxicol 2007;45(11):2179-2205. DOI: 10.1016/j.fct.2007.05.015.

57. Vargas-Rastrepo F, Sabogal-Guáqueta AM, CardonaGómez GP. Quercetin ameliorates inflammation in CA1 hippocampal region in aged triple transgenic Alzheimer's disease mice model. Biomedica 2018;38(0):69-76. DOI: 10.7705/biomedica.v38i0.3761.

58. Aguiar CL. Isoflavonas de soja e propriedades biológicas. Bol Centro Pesqui Process Aliment 2002;20(2):323334.

59. Clapauch R, Meirelles RMR, Julião MASG, Loureiro CKC, Giarodoli PB, Pinheiro AS, Harrigan AR, Spritzer PM, Pardini DP, Weiss RV, Athayde A, Russo LA, Póvoa LC. Fitoestrogênios: Posicionamento do Departamento de Endocrinologia Feminina da Sociedade Brasileira de Endocrinologia e Metabologia (SBEM). Arq Bras Endocrinol Metabol 2002;46(6):679-695. DOI: http://dx.doi.org/10.1590/S0004-27302002000600013.

60. Schmidt M, Arjomand-Wölkart K, Birkhäuser MH, Genazzani AR, Gruber DM, Huber J, Kölbl H, Kreft D, Leodolter S, Linsberger D, Metka M, Simoncini T, Vrabic Dezman L. Consensus: soy isoflavones as a firstline approach to the treatment of menopausal vasomotor complaints. Gynecol Endocrinol 2016;32(6):1-4. DOI: 10.3109/09513590.2016.1152240.

61. Tranche S, Brotons C, Pisa BP, Macías R, Hevia E, Marzo-Castillejo M. Impact of a soy drink on climacteric symptoms: an open-label, crossover, randomized clinical trial. Gynecol Endocrinol 2016;32(6):477-482. DOI: 10.3109/09513590.2015.1132305.

62. Nadadur M, Stanczyk FZ, Tseng CC, Kim $\mathrm{L}$, Wu AH. The Effect of Reduced Dietary Fat and
Soy Supplementation on Circulating Adipocytokines in Postmenopausal Women: A Randomized Controlled 2Month Trial. Nutr Cancer 2016;68(4):554-559. DOI: 10.1080/01635581.2016.1158294.

63. Nahás EAP, Nahás Neto J, Luca LA, Traiman P, Pontes A, Dalben I. Efeitos da Isoflavona Sobre os Sintomas Climatéricos e o Perfil Lipídico na Mulher em Menopausa. Rev Bras Ginecol Obstet 2003;25(5):337-343. DOI: http://dx.doi.org/10.1590/S0100-72032003000500006.

64. Sousa RL, Filizola RG, Diniz MFFM, Sousa ESS, Moraes JLR. Ensaio clínico placebo-controlado com isoflavonas da soja para sintomas depressivos em mulheres no climatério. Rev Bras Ginecol Obstet 2006;28(2):91-100. DOI: http://dx.doi.org/10.1590/S0100-72032006000200004.

65. Jamilian M, Asemi Z. The Effects of Soy Isoflavones on Metabolic Status of Patients With Polycystic Ovary Syndrome. J Clin Endocrinol Metab 2016;101(9):3386-3394. DOI: $10.1210 /$ jc.2016-1762.

66. Esteves EA, Monteiro JBR. Efeitos benéficos das isoflavonas de soja em doenças crônicas. Rev Nutr 2001;14(1):43-53. DOI: http://dx.doi.org/10.1590/S141552732001000100007.

67. Lu C, Wang Y, Wang D, Zhang L, Lv J, Jiang $\mathrm{N}$ et al. Neuroprotective Effects of Soy Isoflavones on Scopolamine-Induced Amnesia in Mice. Nutrients 2018;10(7):E853. DOI: 10.3390/nu10070853.

68. Marques A. Chronic Lyme Disease: An appraisal. Infect Dis Clin North Am 2008;22(2):341-360. DOI: http://dx.doi.org/10.1016\%2Fj.idc.2007.12.011.

69. Smith LJ, Kalhan R, Wise RA, Sugar EA, Lima JJ, Irvin CG, Dozor AJ, Holbrook JT. American Lung Association Asthma Clinical Research Centers. Effect of a Soy Isoflavone Supplement on Lung Function and Clinical Outcomes in Patients With Poorly Controlled Asthma. JAMA 2015;313(20):2033-2043. DOI: 10.1001/jama.2015.5024.

70. Nardi J, Moras PB, Koeppe C, Dallegrave E, Leal MB, Rossato-Grando LG. Prepubertal subchronic exposure to soy milk and glyphosate leads to endocrine disruption. Food Chem Toxicol 2017;100:247-252. DOI: http://dx.doi.org/10.1016/j.fct.2016.12.030. 